\title{
Novas abordagens de criação de materiais para o Design
}

\author{
New Approaches to create Materials for Design
}

\author{
MONTEIRO, Amanda; Mestra; Universidade de São Paulo \\ amandasousamonteiro@usp.br \\ DANTAS, Denise; Doutora; Universidade de São Paulo \\ dedantas@usp.br
}

\section{Resumo}

O presente artigo propõe uma contribuição aos estudos de desenvolvimento de novos materiais dentro da temática "design e materiais". O seu objetivo é elucidar abordagens metodológicas voltadas à criação de materiais levando em consideração a experiência do usuário, procurando incentivar novas pesquisas nesta área ainda pouco estudada no Brasil. Para isso, realizou-se um levantamento bibliográfico que definiu como principal foco de pesquisa as abordagens Material Driven Design de Karana et al. (2015) e DIY materials de Rognoli et al. (2015). Tais abordagens propõem a criação, avaliação e planejamento de produtos tendo como ponto de partida a utilização ou a criação de novos materiais. Os principais aspectos destes estudos foram organizados em uma tabela que pretende auxiliar estudantes e profissionais sobre qual caminho percorrer durante o projeto do produto que tenha como requisito a criação ou a utilização de um material inovador.

Palavras Chave: material driven design; DIY materials; criação de materiais.

\begin{abstract}
This article proposes a contribution to the studies of development of new materials as part of the theme "design and materials". Its objective is to elucidate methodological approach's aimed to creating materials taking into account the user experience, , encouraging new research in this area that is still poorly addressed in Brazil. For this, a bibliographical survey was carried out and was defined as main research focus the Material Driven Design approach by Karana et al. (2015) and DIY materials by Rognoli et al. (2015). Such approaches propose the creation, evaluation and planning of products based on the use or creation of new materials. The main aspects of these studies were organized in a table that intends to help students and professionals during the process of product design that requires the creation or use of an innovative material.
\end{abstract}

Keywords: material driven design; DIY materials; development of materials. 


\section{Introdução}

Durante a história da humanidade, materiais estiveram estreitamente ligados à evolução das práticas e vidas humanas. Estuda-se civilizações antigas através dos vestígios materiais que sobreviveram ao tempo. As novas tecnologias dessas sociedades são tão importantes que até mesmo utiliza-se materiais que impactaram em tais períodos para denominar as eras históricas, como por exemplo, as assim chamadas Idade da Pedra e Idade do Bronze (RAMALHETE, 2012).

A história do Design também é impactada diretamente pela história dos materiais. Na revolução industrial, nos movimentos Art and Crafts, Art Nouveau, De stijl, entre outros, é possível perceber como determinados materiais revolucionaram a cultura material e impactavam diretamente na sociedade e nos meios de produção (BÜRDEK, 1999).

O design industrial é responsável por melhorias em nossa cultura material e ao longo dos anos vem transformando o espaço, o trabalho e também as relações humanas. Ao utilizar recursos como os da maquinaria, dos processos produtivos e materiais, entrega produtos e serviços com maiores qualidades funcionais e estéticas (BONSIEPE, 2012). O designer, com atos projetuais, intervém e supera dificuldades encontradas na realidade da produção industrial, "Afinal, projetar, introduzindo as mudanças necessárias, significa ter a predisposição para mudar a realidade sem se distanciar dela" (BONSIEPE, 2011, p. 37).

Ao se falar de materiais, percebe-se que estes ainda são pouco explorados quanto a sua criação por parte de designers e extensivamente estudados quanto à sua aplicação em projetos. Entretanto, é um campo que permite avanços por transformar tecnologias inovadoras em possibilidades reais aos usuários, podendo ser gerador de novas experiências, comportamentos e arquiteturas (ASHBY; JOHNSON, 2011).

Novas pesquisas demonstram que os designers devem mudar seu posicionamento quanto aos materiais, deixando de ser apenas selecionadores de alternativas para serem geradores e pensadores originais, que, a partir de um diálogo direto com os produtores e engenheiros, possam promover o desenvolvimento e aplicação de materiais (BÜRDEK; EISELE, 2011). Para isso, é necessário uma série de conhecimentos, sejam eles técnicos ou relativos à questões semânticas, que com a abordagem correta, poderá ser absorvido de maneira eficaz por designers de produtos.

Abordagens como o Material Driven Design (MDD), podem ser consideradas uma nova forma de se trabalhar materiais, tendo-os como ponto de partida no processo de design e definindo-se padrões para a criação de produtos baseados na experiência do usuário (KARANA et al., 2015). Outras abordagens, como o DIY Materials proporcionam uma experiência de criação única e de quebra com os paradigmas projetuais. Pode-se então, incentivar e direcionar os profissionais na criação e desenvolvimento de novas propostas, tendo como base os processos criativos pertinentes a área (ROGNOLI et al., 2015).

Elucidar tais abordagens e seus impactos no design de produto foi o principal objetivo do presente artigo, que pretende incentivar novas pesquisas nesta área ainda pouco estudada dentro do design no Brasil. Os principais aspectos destes estudos foram organizados em uma tabela que pretende auxiliar estudantes e profissionais sobre qual caminho percorrer durante o projeto do 
produto que tenha como principal requisito criação ou a utilização de um material inovador.

\title{
2 Design e seleção de materiais
}

De acordo com o WDO (World Design Organization) o design industrial é um processo estratégico de solução de problemas que impulsiona a inovação, constrói o sucesso do negócio e leva a uma melhor qualidade de vida por meio de produtos, sistemas, serviços e experiências inovadores. Preenche a lacuna entre o que não é e o que é possível. Ele conecta inovação, tecnologia, pesquisa, negócios e clientes para fornecer novos valores e vantagens competitivas em esferas econômicas, sociais e ambientais (WDO, 2018).

Os materiais desenvolvem um papel essencial nos projetos de design, pois permitem que se encontre novas formas de usos e significações. Existe uma ampla gama de opções que deve ser atentamente ponderada, visando sempre absorver as melhores características. Essas podem ser físicas, estéticas ou funcionais e em todas as dimensões, denotam uma série de estudos e avaliações para que sanem os requisitos de um projeto (PISELLI et al., 2015).

De acordo com Piselli et al. (2015), pode-se aplicar materiais de diferentes maneiras. A abordagem tradicional (presente na maioria das metodologias projetuais) começa com a introdução aos conceitos fundamentais de engenharia de materiais, que vai desde classes de materiais como as suas propriedades intrínsecas (físico, mecânico, térmico, óptico, etc.), até as questões ambientais, mecanismos de falha, assim como as questões estéticas associadas.

\begin{abstract}
Vários autores incluíram a seleção de materiais nos próprios processos de design que conceberam, quer como uma fase ou um "passo" a percorrer para cumprir uma etapa do processo de design, tal como Munari (1988) e Bonsiepe (1992). Mais tarde Ashby (1999), Johnson \& Ashby 2002) e Van Kesteren (2008), autores ligados ao design e seleção de materiais salientaram a importância dessa escolha fundamentada de materiais como sendo um método paralelo interagindo com o processo de design (RAMALHETE, 2012, p.66).
\end{abstract}

Como mostra Ashby e Johnson (2011), são principalmente inovações na área dos materiais que permitem inovações no design industrial. Tais materiais inspiram, sugerem novas soluções, sensações visuais, táteis, emocionais e esculturais. Quanto as metodologias projetuais, percebe-se que a seleção de materiais e processos de fabricação é uma fase comum e assume um nível estratégico de atuação quanto a conceituação do produto.

Um dos métodos de seleção de materiais mais utilizados é baseado em índices de desempenho e gráficos de propriedades de materiais (ASHBY; JOHNSON, 2011). O método também é implementado como uma ferramenta de software de computador, o "CES-Cambridge Engineering Selector". "No design de produtos, o ato de seleção de materiais envolve a definição de um conjunto de requisitos de design de modo a obter uma lista de materiais e processos viáveis" (RAMALHETE, 2012, p. 71).

Para Edwards (2014), apesar da qualidade tanto da metodologia quanto do software, existe pouco suporte disponível para os requisitos não técnicos, como a estética e outros atributos emocionais, tão importantes para o design de produtos. A dificuldade em se mensurar tais atributos é a principal razão desta lacuna metodológica, já que procedimentos sistemáticos formais não se aplicam. 
A experiência do usuário é muito relevante nesse aspecto, pois acaba sendo decisiva para a aceitação ou não de um determinado produto. Como mostra Karana et al. (2015), a experiência do usuário abrange diversos aspectos relacionados a percepção do mesmo, incluindo a experiência estética, a experiência do significado, a experiência emocional e nível performativo do produto quanto as expectativas do usuário.

Levando em consideração a experiência do usuário, nos últimos anos o assunto tem ganhado espaço em pesquisas dentro da área do design, abordando as questões semânticas dos materiais e também dos processos de seleção dentro do pensamento criativo nas fases iniciais de projeto (BARAUNA et al., 2015).

No processo de design tradicional, a seleção de materiais é um processo que ocorre em um estágio avançado de "desenvolvimento", onde os critérios de seleção de materiais são definidos pelo contexto de fabricação e custos para realizar um conceito de produto já maduro. O contexto nesta fase é muito concreto e decomposto, o que permite selecionar quais materiais ou processos são apropriados e quais não são de uma maneira muito analítica (VAN-BEZOOYEN, 2014, p. 281, tradução nossa).

Segundo Ashby e Johnson (2011), para tomar decisões sobre o uso eficiente de materiais, é acima de tudo, necessário ter uma base sólida de conhecimentos técnicos sobre os materiais e os seus processos produtivos. Tais conhecimentos são gerados a partir de caracterizações que geram dados brutos, que precisam ser destilados por análises estatísticas adequadas que possam ser transformadas em atributos conhecidos como "propriedades do material". Utilizar tais dados de modo seguro, dentro da dimensão de uso real de um produto, é um grande desafio.

Vale ainda ressaltar que a separação entre a engenharia de materiais e o design de produtos gera problemáticas para ambos os lados dentro deste processo. Para os designers, compreender atributos técnicos oriundos da engenharia é de grande dificuldade, enquanto para os engenheiros, o difícil é compreender os projetos e anseios dos designers, de acordo com sua cultura projetual. Os aspectos sensoriais dos materiais são de extrema importância para o design, o que é de difícil compreensão para os engenheiros. Como definido por Del Curto et. al. (2010, p.16, tradução nossa), "as pesquisas sobre a expressividade e a sensorialidade dos materiais tem grande interesse, por enfatizar os aspectos comunicativos de um produto". Nesse sentido, a autora enfatiza a necessidade de ampliação dos processos projetuais das características superficiais dos materiais como uma competência dos designers.

\section{Material Driven Design}

Um novo método ganha cada vez mais espaço nas publicações na área de design e chama a atenção pelas possibilidades que oferece. Karana et al. (2015) propuseram o método Material Driven Design (MDD), que tem o material como ponto de partida dentro de um projeto de design.

O método sugere o que os autores chamam de "uma jornada pelas propriedades materiais e qualidades experienciais para experiência em materiais, desde a visão de experiência de materiais até qualidades experienciais e propriedades materiais e, finalmente, produtos" (KARANA et. al., 2015, p.49, tradução nossa). Para isso, as atividades de projeto são organizadas em quatro etapas principais que trabalham o material desde sua concepção até a experiência do usuário. 
$\mathrm{Na}$ primeira etapa, procura-se compreender o material. Para isso é realizada a caracterização técnica e experiencial deste material. Nos dias de hoje, muitos materiais já possuem caracterizações completas disponíveis tanto em publicações acadêmicas quanto em bibliotecas de materiais. É importante então, saber quais aspectos são mais importantes para o projeto e a melhor forma de traduzir os mesmos como requisitos de projeto.

A segunda etapa visa criar uma visão para a experiência do material, para isso, reúne uma diversificada gama de pessoas para explorar o material. Podem ser realizadas oficinas, grupos focais e outras formas que permitam que o usuário vivencie o material e interaja com ele.

A terceira etapa se baseia em manifestar os padrões da experiência do material, ou seja, traduzir o resultado da segunda etapa em atributos reais e tangíveis que possam ser transformados e aplicados em um produto real. Por último, na quarta etapa, cabe ao designer projetar com o material e criar conceitos de produto que melhorar traduzam todos os pontos levantados nos padrões de experiência do usuário.

Os autores sustentam que o método pode auxiliar designers a adquirir competências na exploração, compreensão, definição e mobilização de propriedades materiais únicas e qualidades experienciais no design. Dessa forma, a problemática existente na seleção de materiais, que diz respeito aos atributos não técnicos, seria solucionada. De acordo com Karana et al. (2008), o ponto chave é a experiência do usuário, que se refere às experiências que as pessoas têm com e através dos materiais de um produto. Na Figura 1 é possível observar o resultado de uma oficina de materiais onde trabalhou-se com compósitos de resíduos de café.

Figura 1 - Resultado de oficina de materiais onde trabalhou-se o MDD para a criação de um material compósito

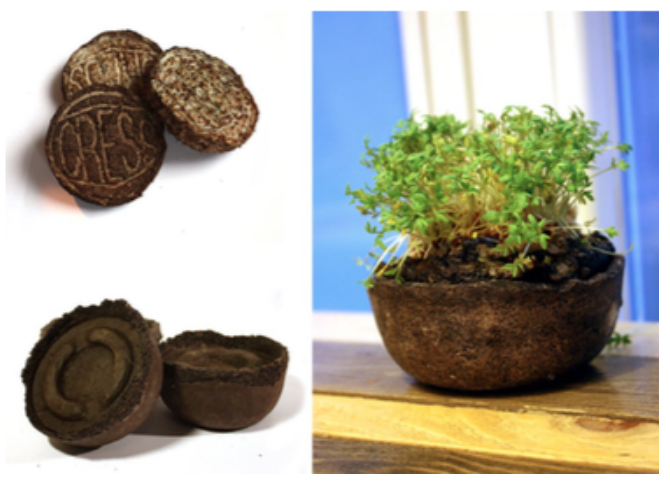

Fonte: Karana et al. (2015).

Van-Bezooyen (2014) também aborda esta nova forma de concepção, que sugere através de etapas interativas a confecção, teste e avaliação de protótipos de materiais, podendo envolver o usuário na experiência desde o início do processo de design. Segundo o autor, a manufatura é crucial no aspecto dos materiais. Pensar em materiais que tenham ótimos atributos técnicos, excelentes aplicações e que por outro lado demandem processos produtivos extremamente poluentes ou de alto custo econômico, tornam o mesmo inviável. Assim, é fundamental encontrar soluções que abranjam todos esses aspectos.

Manzini (2008) defende que designers possuem a capacidade de orientar estrategicamente 
às próprias atividades, ou seja, possuem a habilidade de definir objetivos que combinem necessidades do projeto com critérios da sustentabilidade. O autor também afirma que o desenvolvimento de materiais é uma atividade coerente e convergente as necessidades de nossa sociedade, "Hoje em dia, é forçoso partimos do princípio de que a investigação de materiais é, por direito próprio, um campo possível do design."(MANZINI, 1993, p.43).

\section{DIY Materials}

Na contramão aos avanços das tecnologias de produção em massa, surgem alternativas, como os chamados materiais faça-você-mesmo (DIY Materials). Essa nova abordagem traz uma nova dimensão para o relacionamento entre designers, tecnologias, processos de produção e materiais.

Os DIY Materials podem ser produzidos individualmente ou coletivamente através de oficinais de trabalho de materiais e de técnicas desenvolvidas pelos próprios designers (ROGNOLI et al., 2015). Na Figura 2 é exposta a produção de um compósito de fibra natural biodegradável feito de sobras de produção de madeira e café, por Nikolaj Steenfatt (ROGNOLI et al., 2015).

Figura 2 - Produção através do método DIY materials.
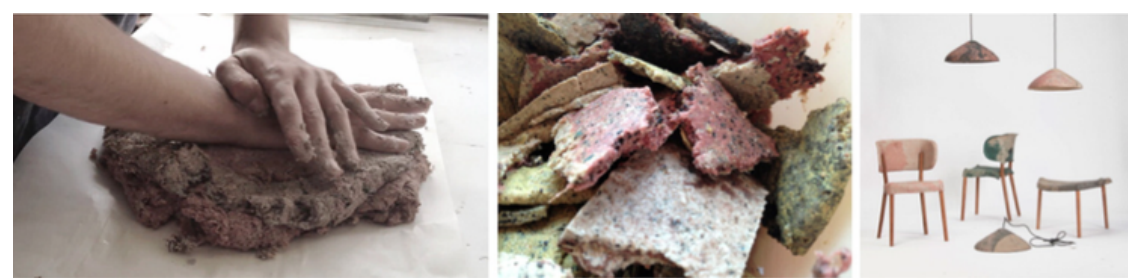

Fonte: Rognoli et al. (2015).

O método funciona como uma sistematização das habilidades pessoais de criação, munida das tecnologias de manufatura digitais. Os DIY materials surgem em um contexto de forte demanda por personalização. Tais materiais oferecem uma oportunidade para reconsiderar as características dos processos de fabricação existentes e das propriedades do material industrializado (ROGNOLI et al., 2015).

A autoprodução refere-se a uma maneira de controlar os processos de produção por meio de experimentação e ajustes: criar um material em um "laboratório" e no mesmo laboratório, processando o material em um produto. Essa abordagem faz com que o projetista veja quase tudo como possível candidato como uma questão de esforço de autoprodução (ROGNOLI et al., p. 693, 2015, tradução nossa).

Na criação de materiais seguindo o DIY Materials, designers e criadores são influenciados pela forma intercambiável dos materiais. Por meio do "aprender fazendo" e "aprender interagindo" podem se expressar criando materiais e produtos exclusivos que possam revelar suas personalidades. 


\section{Discussão}

Existem diversas lacunas quanto aos aspectos relacionados diretamente aos materiais e seus significados, no design de produtos. Quando se fala da experiência com o material, esforços vem sendo apreendidos, principalmente no que diz respeito a compreensão do mesmo (WILKES et al., 2016; GIACCARDI; KARANA, 2015).

No Brasil, entretanto, o cenário ainda é embrionário. Novos materiais são criados, principalmente por engenheiros, porém poucas são as aplicações efetivas destes materiais no design. Por parte dos designers, podem ser encontrados excelentes exemplos na literatura, como os módulos para plantas composto por um compósito de resíduo de fibra de vidro (ALMEIDA; SOUZA, 2016); Compósito polimérico reforçado por resíduo resultante do processo de fabricação da cerveja com aplicação na produção de corpo de uma guitarra (OLIVEIRA; NOVAES, 2016) e o piso parquet de um compósito com fibra de tururi (OLIVEIRA, 2011), entre outros.

Todavia, quanto a experiência a experiência com o material, muito pouco é encontrado. As duas abordagens apresentadas demonstram a importância de incentivar o desenvolvimento de novos materiais, conhecendo as suas propriedades e seu comportamento como produto através. A seguir, serão apresentados aspectos sistematizados das mesmas assim como vantagens/desvantagens de seus usos. Na Tabela 1, aborda-se o Material Driven Design de Karana et al. (2015).

Tabela 1 - Sistematização do Material Driven Design

Práticas projetuais coletivas, para Viabilizar criação com materiais apoiar designers na estruturação, comunicação e reflexão sobre suas ações no design para experiências com materiais. totalmente novos e sem parâmetros estéticos e sensoriais definidos. Liberdade criativa.
Ausência de ferramentas ou estratégias que possam apoiar a exploração das características desconhecidas desses novos materiais. Risco de projetar um produto que possa preencher as capacidades funcionais de um novo material sem criar um valor real para a sociedade

Fonte: o autor.

Entre as vantagens e as desvantagens apresentadas, destaca-se a dificuldade em se criar um valor real para a sociedade. Este é um ponto que, somente através do constante aprimoramento das abordagens, poderá ser alcançado. As expectativas do usuário, baseadas nos múltiplos aspectos citados anteriormente, ainda é de difícil acesso, entretanto, passível de ser melhor compreendida e alcançada. Na Tabela 2, DIY Materials de Rognoli et al. (2015) é sistematizado e comparado. 
Tabela 2 - Sistematização do DIY Materials

Práticas de autoprodução individual ou coletiva, baseadas em técnicas e processos da própria invenção do designer.
Exploração das habilidades de criação desenvolvendo as ferramentas e máquinas necessárias, controlando todos os aspectos da produção, como um artesão.
Ausência de ferramentas ou estratégias que possam apoiar os projetistas na exploração e compreensão das características desconhecidas desses novos materiais. Dificuldade de replicação do material. Pouco impacto na produção industrial em geral.

Fonte: o autor.

A partir da apresentação das duas abordagens é possível fazer questionamentos sobre a aplicabilidade das mesmas no Design Industrial e possíveis caminhos a serem percorridos para driblar as desvantagens conhecidas. É muito importante, desde já, pensar metodologias que deem suporte e permitam a sua verificação e replicação. Como dito anteriormente, o designer deve cambiar seu posicionamento e se tornar um ator ativo nestas novas configurações. Deve procurar a melhor forma para que profissionais e usuários possam participar ativamente da criação de novos materiais e que principalmente, suas necessidades sejam traduzidas em projetos coerentes e passíveis de fabricação.

\section{Conclusões}

Os estudos de novas abordagens de criação de materiais levando em conta aspectos da experiência com o material ainda é um campo novo e que carece de maior aprofundamento. Pensar em metodologias que possam dar suporte e auxiliar na sua concretização é de extrema importância. Acredita-se que problemáticas que surgem no desenvolvimento e aplicação de novos materiais em projetos de design decorrem das dificuldades em se compreender aspectos técnicos dos mesmos, por caracterizações incompletas e também pela dificuldade existente em relação às expectativas e compreensões do novo material quanto aos atributos não técnicos, principalmente no que diz respeito a experiência do usuário e aspectos sensoriais. Espera-se gerar novos estudos e possibilidades metodológicas que sanem, assim, essas dificuldades.

\section{Referências}

ALMEIDA, C. E. S.; SOUZA, C. F. de. Revestimentos para Superfícies Verticais a partir de Resíduos de PRFV: Plástico Reforçado de Fibra de Vidro. Revista D.: Design, Educação, Sociedade e Sustentabilidade, Porto Alegre, v.8 n.2, 20-37, 2016.

ASHBY, M. F.; JOHNSON, K. Materiais e design: Arte e ciência da seleção de materiais no design de produto. Rio de Janeiro: Elsevier, 2011.

BARAUNA. D, RAZERAB. D. L., HEEMANNB, A. Seleção de Materiais no Design: Informações 13o Congresso Brasileiro de Pesquisa e Desenvolvimento em Design, Univille, Joinville (SC) 
Necessárias ao Designer na Tomada de Decisão para a Conceituação do Produto. Design \& Tecnologia, v.5, n.10, 1-9, 2015.

BONSIEPE, G. Design, Cultura e Sociedade. São Paulo, SP: Blucher, 2011.

Design: como prática de projeto. São Paulo, SP: Blucher, 2012.

BÜRDEK, B. E. Design - História, teoria e prática do design de produtos. São Paulo: Editora Edgard Blücher Ltda., 1999. 496 p.

BÜRDEK, B.E., EISELE, P. Design, Anfang des 21. Jahrhunderts: Diskurse und Perspektiven. Germany: Av Edition Gmbh, 2011.

DEL CURTO et. al. La pelle del design. Progettare la sensorialità. Milano: Lupetti, 2010.

EDWARDS, K. Interaction between Functional and Human-Centered Attributes in Materials Selection. In: KARANA, E., PEDGLEY, O., \& ROGNOLI, V. (Ed.). Materials experience: Fundamentals of materials and design. Oxford, UK: Butterworth-Heinemann. 2014. p. 287-299.

GIACCARDI, E.; KARANA, E. Foundations of Materials Experience: An Approach for $\mathrm{HCl}$. In: Proceedings of the 33rd SIGCHI Conference on Human Factors in Computing Systems., 2015, Nova York: ACM. p. 2447-2456.

KANDACHAR, P. Materials and Social Sustainability. In: KARANA, E., PEDGLEY, O., \& ROGNOLI, V. (Ed.). Materials experience: Fundamentals of materials and design. Oxford, UK: ButterworthHeinemann. 2014. p. 91-104.

KARANA, E., HEKKERT, P., KANDACHAR, P., Materials experience: descriptive categories in material appraisals. In: International Conference on Tools and Methods in Competitive Engineering. Izmir, 2008.

KARANA, E.,BARATI, B., ROGNOLI, V., VA-DER-LAAN, A. Z. Material Driven Design (MDD): A Method to Design for Material Experiences. International Journal of Design, vol. 9, n.2, p. 35-54, 2015.

MANZINI, E. A Matéria da Invenção. Lisboa: Centro Português de Design, 1993.

. Design para a inovação social e sustentabilidade: comunidades criativas, organizações colaborativas e novas redes projetuais. Rio de Janeiro: E-papers, 2008. 104 p.

OLIVEIRA, A. K. F.; NOVAES, R. Guitarra de compósito polimérico vegetal: Um estudo sobre novos materiais no design de produtos. Revista D.: Design, Educação, Sociedade e Sustentabilidade, Porto Alegre, v. 8 n.2, 125-141, 2016. 
PISELLI, A., GARBAGNOLI, P., DEL-CURTO, B. Innovative light shapes: an educational experience on materials and manufacturing technologies' selection tools. In: Proceedings of INTED2015 Conference, 2015.

RAMALHETE, P. M. B. S. Metodologia de seleção de materiais em design: base de dados nacional. 2012. 315 f. Tese (Doutorado em Design) - Departamento de Comunicação e Arte, Universidade de Aveiro, Aveiro, 2012.

ROGNOLI, V., BIANCHINI, M., MAFFEI, S., KARANA, E. DIY materials. Materials and Design, v.86, p. 692-702, 2015.

VAN-BEZOOYEN, A. Materials Driven Design. In: KARANA, E., PEDGLEY, O., \& ROGNOLI, V. (Eds.). Materials experience: Fundamentals of materials and design. Oxford, UK: ButterworthHeinemann. 2014. capítulo 20, p. 277-286.

WDO - World Design Organization. Definition of Design. Disponível em:

<http://wdo.org/about/definition/>. Acesso em: 05 abr. 2018.

Wilkes, S.; Wongsriruksab, S.; Howesc, P.; Gamestera, R.; Witcheld, H.; Conreene, M.; Laughlina, Z.;Miodownika, M. Design tools for interdisciplinary translation of material experiences. Materials and Design, v.90, p. 1228-1237, 2016. 